\title{
Blocking immune intrusion into the brain suppresses epilepsy in Rasmussen's encephalitis model
}

\author{
Lawrence Steinman
}

Departments of Pediatrics and Neurology and Neurological Sciences, Stanford University, Stanford, California, USA.

\author{
Rasmussen's encephalitis (RE) is a neuroinflammatory disease that typically \\ affects only one hemisphere of the brain, resulting in severe seizures. Sixty \\ years after the disease was first described, the preferred and best treatment \\ option for RE is grotesque and involves removing a hemisphere of the \\ brain (hemispherectomy); therefore, a better understanding of this seizure \\ disorder may provide additional, less invasive therapeutic options. In this \\ issue of the $\mathrm{JCl}$, Carmant and colleagues have developed an animal model of \\ this focal seizure disorder. The model provides experimental insights into \\ the pathogenesis of RE and potential new treatments for this disease.
}

\section{A mouse model of RE}

In this issue, a research team composed of medical scientists from the fields of neuroimmunology, neuropathology, and pediatric immunology collaborated to develop an animal model of RE (5). Carmant and colleagues transferred human peripheral blood lymphoid cells from patients with $\mathrm{RE}$ into NOD-Scid IL-2 $\gamma \mathrm{c}$-null (NSG) mice $(6,7)$. Following engraftment of REderived lymphocytes, the mice developed severe seizures, and pathology analysis revealed the presence of human $\mathrm{T}$ cells in the perivascular cuffs, similar to what is seen in patients with RE. These T cells found in the brain of these mice produced a key inflammatory cytokine, IFN- $\gamma$, and a key mediator of cytotoxicity, granzyme $\mathrm{B}$.

The damage in the brains of the $\mathrm{RE}$ mouse model was bilateral, involving both hemispheres, a feature that is distinctly different from what is seen in humans with RE. Rodent and human brains are different in at least one major respect: unlike humans, mice do not have a dominant hemisphere. At least in this model, there was no bias in pathology that was restricted to one hemisphere. Whether the bilateral manifestation in the RE mice has anything to do with cerebral dominance is mere conjecture at this point; however, there is evidence (reviewed in ref. 8) that handedness, particularly left handedness, correlates with autoimmunity within families.

It remains unresolved as to why the human disease has pathology limited to one hemisphere. Viral encephalitis often begins with a single focal lesion; however, with the exception of RE, there is little evidence of such widespread damage restricted to a single hemisphere in any other type of viral encephalitis or any other kind of neurodegenerative disease (1-4). Perhaps, the closest approximation to what is observed in RE is the case for herpes simplex virus (HSV) encephalitis, which often starts in one temporal lobe but does not progress to envelop and destroy the whole hemisphere. Post-in- 


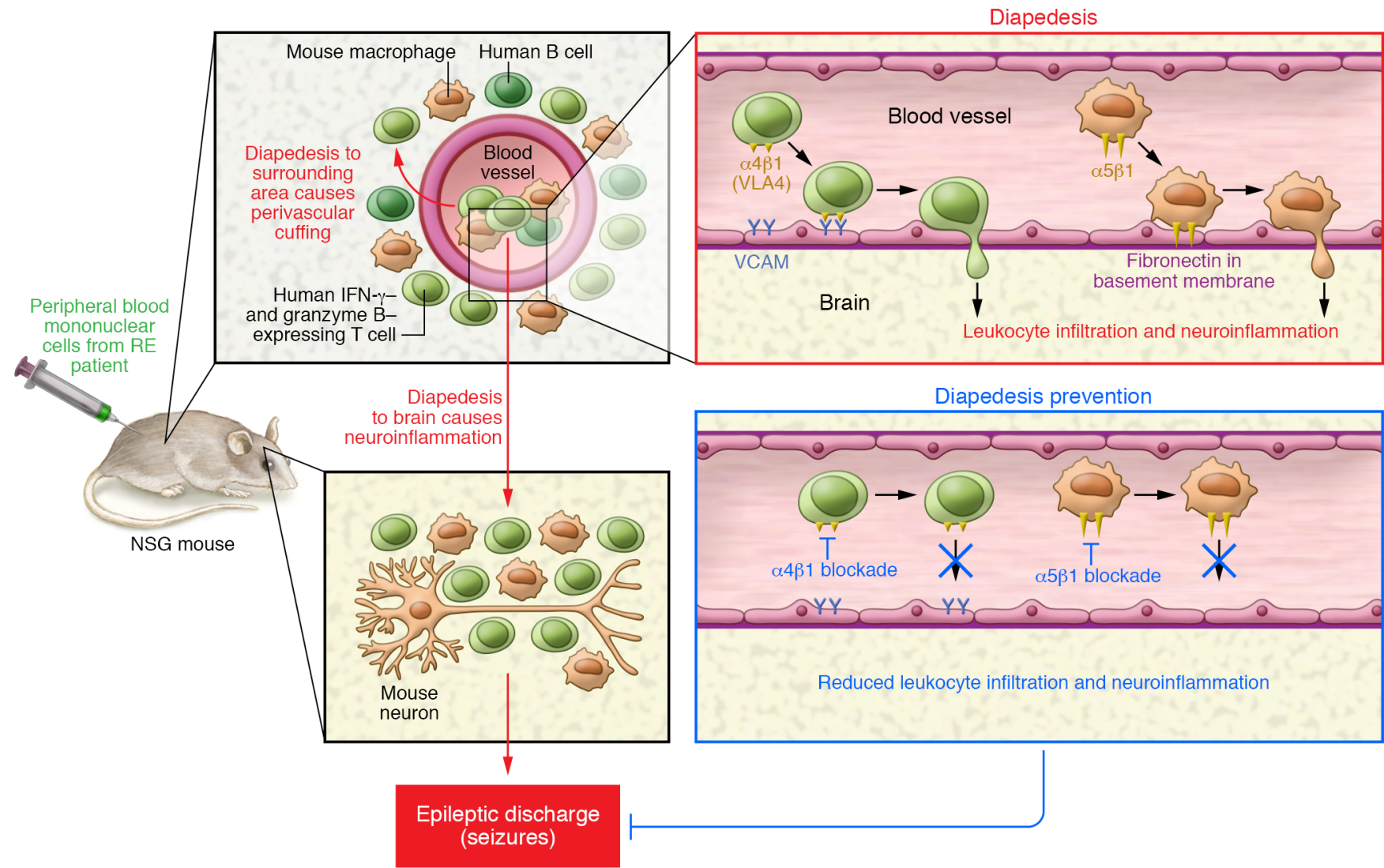

Figure 1. A mouse model of RE. In this issue, Carmant and colleagues describe the development of a mouse model of RE, in which peripheral blood mononuclear cells from RE patients are transferred into NSG mice. These RE mice develop seizures following engraftment and exhibit several other hallmarks of RE, including perivascular cuffing and neuroinflammation. Neuroinflammation is linked to diapedesis of $T$ cells, which is mediated by the interaction of $\alpha 4 \beta 1$ on $T$ cells with VCAM on the vascular endothelium, and macrophages, via the interaction of macrophage $\alpha 65$ and fibronectin in the basement membrane. Blocking either $\alpha 4 \beta 1$ or $\alpha 5 \beta 1$ reduced seizure in this model. Moreover, this model will allow further exploration of potential therapeutic strategies for RE.

fectious encephalomyelitis and acute disseminated encephalomyelitis (ADEM), along with classical animal models, collectively known as experimental autoimmune encephalomyelitis (EAE), all generally involve both hemispheres. One furtherfactor that must be considered in the etiology of RE is that in some patients, there are areas of focal cortical dysplasia and tuberous sclerosis with inflammatory and gliotic pathology. The question of whether these dysplastic areas might serve as a nidus for an inflammatory response has been raised (2). A combination of a viral and dysplastic etiology might also be in play as a possibility.

Another unsolved issue in $\mathrm{RE}$ is whether the adaptive immune response underlying $\mathrm{T}$ cell- and antibody-based immunity is driven by a particular antigen on neurons. Although there is evidence of adaptive immunity to glutamate receptors $(9,10)$, immune responses to glutamate receptors are seen in a variety of inflam- matory epilepsies. It is now well established that one of the common triggers of epilepsy is an inflammatory response to one or more brain antigens. Molecular mimicry between viruses and components of the CNS is one of the potential drivers of autoimmune inflammation and seizures (11). The known list of antigens associated with immune-mediated epilepsy is large, and commercial tests are now available to detect such immune responses to brain antigens. The evaluation of epilepsy on the pediatric neurology ward now routinely involves a screen from a panel of antibodies obtained from both the serum of peripheral blood and from cerebrospinal fluid. At present, it remains unknown as to whether there is a particular antigen that drives $\mathrm{RE}$ and, if so, what antigen mediates an adaptive immune response.

An important feature of this animal model of RE is the potential to test new approaches for therapy (5). Natalizumab, a humanized antibody targeting $\alpha 4$ integrin
(12), has been one of the most potent drugs approved for both multiple sclerosis and inflammatory bowel disease $(12,13)$. In the RE model, administration of an antibody against $\alpha 4$ integrin reduced the inflammatory infiltrate when given either prophylactically or after the onset of seizures. Both prophylactic and therapeutic treatments induced a shift in the ratio of human $\mathrm{CD} 4^{+}$ to $\mathrm{CD}^{+} \mathrm{T}$ cells infiltrating the brain and, in particular, reduced the number of granzyme $\mathrm{B}^{+}$human $\mathrm{CD}^{+} \mathrm{T}$ cells in the brain. Other natalizumab-associated changes included a reduction in human $\mathrm{CD} 45^{+}$cells, a reduction in glial fibrillary acidic proteinexpressing (GFAP-expressing) astrocytes, and a reduction in HLA-DR ${ }^{+}$cells (5). Video EEG monitoring also showed a reduction in seizures when natalizumab was given prophylactically, though a similar effect was not seen when the antibody was given after seizures were already apparent.

Perivascular cuffs are a prominent feature of neuroinflammation, inflammatory 
epilepsy, multiple sclerosis, ADEM, and EAE and were present in the animal model of RE (ref. 5 and Figure 1). Lymphocyte homing to the CNS involves $\alpha 4 \beta 1$ integrin. Blockade of this integrin drastically reduces the homing of $\mathrm{T}$ cells and $\mathrm{B}$ cells to the brain and ameliorates the frequency of relapses in multiple sclerosis, while delaying disability (13). Blockade of $\alpha 4 \beta 1$ integrin is unfortunately also associated with a reduction in immune surveillance, and over 700 cases of progressive multifocal leukoencephalopathy (PML) have been reported in the 170,000 patients who have taken natalizumab. Most of cases of PML occurred after exposure to $\alpha 4 \beta 1$ integrin blockade for more than a year (14); therefore, long-term application of natalizumab may be problematic in RE, though administration of the antibody early in the course of disease is worthy of further investigation. Coadministration of natalizumab and antiepileptic drugs was reported to be effective in a single case study of RE (15).

The impact of autoimmunity on the homing of T cells, B cells, and macrophages to the brain and other organs is highly nuanced. Whereas T cell and B cell homing to the CNS involves $\alpha 4 \beta 1$ integrin and its receptor VCAM on inflamed endothelium, homing to the intestines involves a different integrin pairing. In inflammatory bowel disease, the heterodimer $\alpha 4 \beta 7$ integrin is recognized by a different receptor, MAdCAM-1, which serves as the key homing molecule (16). Macrophages are also key cellular players in neuroinflammation. $\alpha 5 \beta 1$ integrin is expressed on macrophages that home to the CNS in EAE (17). $\alpha 5 \beta 1$ integrin binds fibronectin (ref. 17 and Figure 1), and treatment of EAE with an antibody against $\alpha 5 \beta 1$ integrin reversed clinical paralysis after encephalomyelitis was present and prevented the onset of disease when given prophylactically (17). Such treatments with an antibody against $\alpha 5 \beta 1$ integrin might permit the homing of $\mathrm{T}$ and $\mathrm{B}$ cells to the brain, thus potentially allowing for immune surveillance and reducing the risk of PML (14) in diseases like RE.

\section{Conclusions}

The team of medical scientists who developed this intriguing model of RE have now opened an area of research into effective new therapies for this disorder. Half a century after Rasmussen and colleagues discovered this horrible disease, perhaps at some point hemispherectomy can be retired to the "Museum of Past and Abandoned Therapies." An example of a therapy in such a theoretical museum might be Halsted's radical mastectomy to treat breast cancer. As Murkherjee so elegantly described in his monograph, "The Emperor of All Maladies" (18), this disfiguring therapy has now been abandoned. For many diseases, we might look at entrants on display in such a museum with wonder and dread, astonished that such therapeutic interventions were even tried. Every medical and surgical specialty can name some therapies that evidence-based medicine has shown to have actually even worked, yet were still dreadful. No therapy may be more dreadful, however, than removing half a brain to stop a seizure disorder. The words grotesque and dreadful used in this Commentary may not be adequate to describe this massive operation, which actually provides great benefit but at a significant cost. I imagine my colleagues in neurosurgery would agree and would hope that the massive surgical procedure could be replaced by a medical therapy someday, leaving hemispherectomy as a historical display in the "Museum of Abandoned Therapies."

Address correspondence to: Lawrence Steinman, Departments of Pediatrics and Neurology and Neurological Sciences, Stanford University, Beckman Center, B002, Stanford, California 94305, USA. Phone: 650.725. 6401; Email: steinman@stanford.edu.
1. Rasmussen T, Olszewski J, Lloydsmith D. Focal seizures due to chronic localized encephalitis. Neurology. 1958;8(6):435-445.

2. Varadkar S, et al. Rasmussen's encephalitis: clinical features, pathobiology, and treatment advances. Lancet Neurol. 2014;13(2):195-205.

3. Bien CG, Schramm J. Treatment of Rasmussen encephalitis half a century after its initial description: promising prospects and a dilemma. Epilepsy Res. 2009;86(2-3):101-112.

4. Bien CG, et al. The natural history of Rasmussen's encephalitis. Brain. 2002;125(pt 8):1751-1759.

5. Kebir H, et al.Humanized mouse model of Rasmussen's encephalitis supports the immune-mediated hypothesis. J Clin Invest. 2018;128(5):2000-2009.

6. Brehm MA, Garcia-Martinez JV, Greiner DL. Humanized mice for immune system investigation: progress, promise and challenges. Nat Rev Immunol. 2012;12(11):786-798.

7. Gregoire-Gauthier J, et al. Use of immunoglobulins in the prevention of GvHD in a xenogeneic NOD/SCID/ $\gamma \mathrm{c}$ - mouse model. Bone Marrow Transplant. 2012;47(3):439-450.

8. Morfit NS, Weekes NY. Handedness and immune function. Brain Cogn. 2001;46 (1-2):209-213.

9. Rogers SW, et al. Autoantibodies to glutamate receptor GluR3 in Rasmussen's encephalitis. Science. 1994;265(5172):648-651.

10. Wiendl $\mathrm{H}$, et al. GluR3 antibodies: prevalence in focal epilepsy but no specificity for Rasmussen's encephalitis. Neurology. 2001;57(8):1511-1514.

11. Steinman L. Autoimmune disease. Sci Am. 1993;269(3):106-114.

12. Rudick R, Polman C, Clifford D, Miller D, Steinman L. Natalizumab bench to bedside and beyond. JAMA Neurol. 2013;70(2):172-182.

13. Polman $\mathrm{CH}$, et al. A randomized, placebo-controlled trial of natalizumab for relapsing multiple sclerosis. N Engl JMed.2006;354(9):899-910.

14. Gorelik L, et al. Anti-JC virus antibodies: implications for PML risk stratification. Ann Neurol. 2010;68(3):295-303.

15. Bittner S, Simon OJ, Göbel K, Bien CG, Meuth SG, Wiendl H. Rasmussen encephalitis treated with natalizumab. Neurology. 2013;81(4):395-397.

16. Berlin C, et al. $\alpha 4 \beta 7$ Integrin mediates lymphocyte binding to the mucosal vascular addressin MAdCAM-1. Cell. 1993;74(1):185-195.

17. Ajami B, et al. Single cell mass cytometry reveals distinct populations of brain myeloid cells in mouse models of neuroinflammatory and neurodegenerative diseases [published online ahead of print March 5, 2018]. Nat Neurosci. https://doi.org/10.1038/s41593-018-0100-x.

18. Murkherjee S. The Emperor of All Maladies. New York, New York, USA: Scribner and Sons; 2010. 